\title{
WIND BARRIER PROTECTION OF A BUILDING ON TOP OF A HILL
}

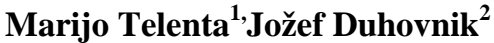 \\ ${ }^{1}$ Faculty of Mechanical Engineering, Aškerčeva 6, 1000 Ljubljana, telenta@lecad.si \\ ${ }^{2}$ Faculty of Mechanical Engineering, Aškerčeva 6, 1000 Ljubljana, joze.duhovnik@lecad.si
}

Keywords: wind barrier; hill; RANS simulation

\begin{abstract}
Numerical simulation is utilized to analyze the velocity profile around a building on top of a hill. The influence of different barrier configurations on the wind velocity around the building is analyzed. A three-dimensional model of a hill, building and barrier was created in SolidWorks, the grid was generated with CutCell Mesher in Ansys, and Ansys FLUENT was used as a solver. The turbulence modelused in the numerical simulation is the Shear Stress Ttransport (SST) $k=\omega$ model. The goal of this paper is to examine the wind barrier effect of various wind barrier configurations on the structure for different wind directions and to select an appropriate wind barrier. An appropriate barrier yields acceptable wind velocities in thedisembarkation area. The results show that all barrier configurations produce lower wind velocity in the disembarkation area of the building. The second and third barrier configurations gave the lowest wind velocities. In addition, the last barrier configuration also satisfies the barrier wind load criterion.
\end{abstract}

\section{INTRODUCTION}

Computational Fluid Dynamics (CFD) simulation offers prediction of wind behavior around objects. In this paper, wind behavior around a building situated on top of a hillis analyzed.The building represents a ski lift. Wind velocity directly influences the comfort of the skiers in the disembarkation area of the lift. The lift has to be functional in high wind conditions. However, wind velocities can reach up to $40 \mathrm{~m} / \mathrm{s}$. These velocities obstruct the normal functioning of the lift, especially in thedisembarkation area. In order to minimize the wind effect, wind barriers are introduced.

Various wind barrier structures are examined and analyzed. Lower wind velocity in the disembarkation area is the criteria for choosing the appropriate barrier. Numerical simulation is utilized since it offers the ability to examine the wind barrier effect for different wind directions. Some previous work with CFD 3D simulations of flow over a hill was done.Previous works (N. N. Sorensen et al. [1], Andreas Bechmann [2], Jackson P.S., Hunt J.C.R. [3], Castro and Palma [4], and Berge et al. [5]) have addressed the problem of wind flow prediction over complex terrain, and wind profile has been addressed by Pingzhiet al. [6]. Also, experimental measurements and numerical simulations have been conducted in order to understand wind flow over a hill (N. N. Sorensen et al. [1]) and around buildings (T. K. Guha et al. [7]).

The goal of this paper is to examine the wind barrier effect of various barrier configurations on the structure for different wind directions and to select an appropriate wind barrier. An appropriate barrier will yield acceptable wind velocities in the disembarkation area.

There are two barrier configurations. The first configuration consists of a single barrier, and the second configuration consists of two barriers. In the case of two barriers, there is a bigger 
front barrier and smaller inner barrier. The hill, the structure and the wind barrier are considered in the simulation. The main objective in the this work is to determine the influence of the barrier on the wind velocity profile in the disembarkation area.

\section{PROBLEM DESCRIPTION}

Three-dimensional CFD simulation was utilized to analyze the wind velocity profile over the hill and around the building. The model of the hill and the building is shown in Fig. 1.

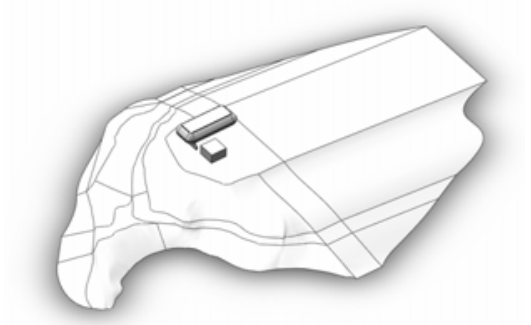

Figure 1: The model of the hill and the building

The building is situated on top of a hill (Fig.2). It represents a ski lift onto which skiers board and from which they disembark. The wind velocity in the disembarkation area can be high. Therefore, the goal of erecting a barrier is to lower the wind velocity in the disembarkation area of the ski lift (Fig. 3).

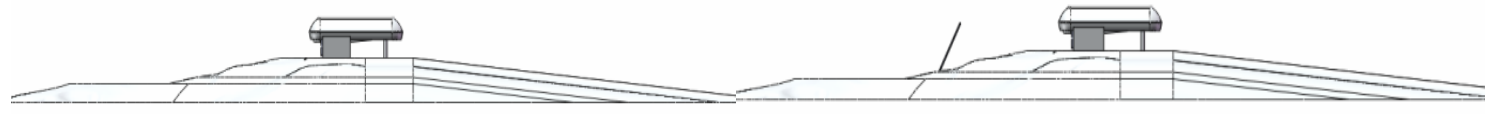

Figure 2: The building on top of the hill

Figure 3: The building and the barrier on top of the hill

Four cases with different wind angles of attack are studied (Fig.4). The first represents a headwind. The other three directions are $30^{\circ}$ left, right and up with respect to the first wind direction. This method captures the wind conditions most likely to be experienced in the vicinity of the building during the winter period.
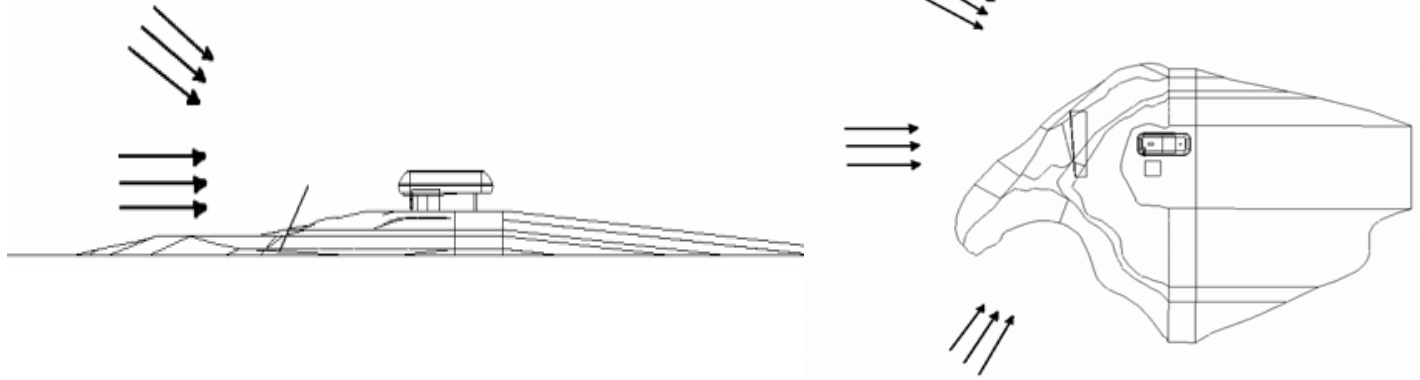

Figure 4: Wind angles of attack 


\subsection{BOUNDARY CONDITIONS}

The computational domain size is: $\mathrm{x}=1350 \mathrm{~m}, \mathrm{y}=300 \mathrm{~m}$, and $\mathrm{z}=750 \mathrm{~m}$ (Fig. 5). The inlet is four hill lengths in front of the hill and the flow outlet is four hill lengths behind the hill. The domain width is five hill lengths and the domain height is two hill lengths.

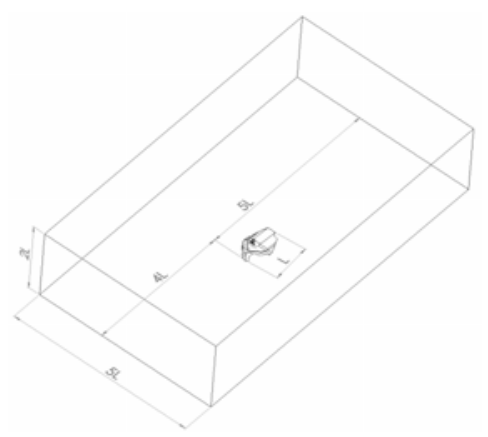

Figure 5: Computational domain

The inlet boundary condition was used for the upstream front face and for the top face of the computational domain. The pressure outlet boundary condition was used for the outlet. The slipwall boundary condition was used forthe side faces. Finally, all other faces were set according to theno-slip wall boundary condition. A uniform velocity profile of $40(\mathrm{~m} / \mathrm{s})$ wasspecified at the inlet.

\subsection{COMPUTATIONAL GRID}

The computational grid is important for CFD simulation and data accuracy. The gridwas created in Ansys ICEM CFD and consists of 7 million elements (Fig.6). The grid was aligned with the flow direction in order to minimize the numerical diffusion. Minimum cell size is $100 \mathrm{~mm}$, maximum size is $25.6 \mathrm{~m}$, and growth rate of the cell is 1.10 . Benefit of the CutCell meshing is that it creates large number of hexahedral elements on complex geometry. Also, the grid dependence was done with previous computational runs.

\subsection{GOVERNING EQUATIONS}

Fluid flow is described by incompressible Navier-Stokes equations:

$$
\begin{aligned}
& \frac{\partial u_{i}}{\partial t}+w_{j} \frac{\partial u_{i}}{\partial x_{j}}=-\frac{1}{\partial \partial} \frac{\partial p}{\partial x_{i}}+v \bar{v}^{2} w_{i}=i=1,2,3 \\
& \frac{\partial u_{i}}{\partial x_{i}}=0
\end{aligned}
$$

where $u_{i}$ is the i-component of the velocity, $x_{i}$ is the i-direction, $\mathrm{t}$ is the time, $\mathrm{p}$ is the pressure, $\rho$ is the density and $v$ is the kinematic viscosity. Eq. 1 represents the momentum equations, and Eq. 2 represents the continuity equation.

\subsection{SOLVER}


The commercial CFD code Ansys Fluent 13.0 was used to solve the equations of fluid motion. Ansys Fluent is general-purpose, proprietary software that is well-known and a standard flow solver. It uses cell-centered numerics, in this simulation, a segregated approach, on a collocated, unstructured grid. The three-dimensional finite volume method was used for RANS equation discretization. The second-order accurate central discretization for the diffusion terms, the second-order accurate upwind discretization for the advection terms, and the second order accurate time discretization were used. The standard algorithm was applied for pressure-velocity coupling. High performance computing (HPC) was utilized for numerical simulations at Faculty of Mechanical Engineering in Ljubljana.

(a)

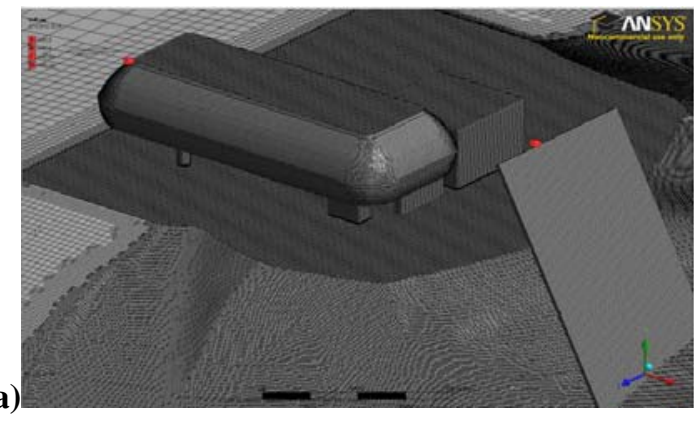

(b)

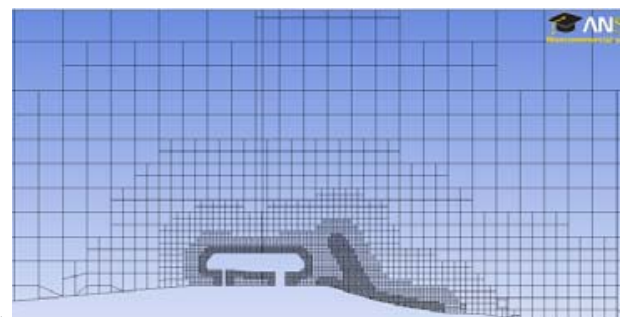

Figure 6: Computational grid: (a) hill, building, and barrier; (b) benchmark mesh; (c) denser mesh

\subsection{TURBULENCE MODELING}

The governing equations due to the Reynolds averaging procedure have additional unknown variables, known as Reynolds stresses. Thus, the governing equations need a turbulence model to be solved. The turbulence model used is the shear stress transport (SST) k- $-\omega$. The kmodel is a two-equation eddy-viscosity model where the Reynolds stresses are linearly related to the mean velocity gradient (Boussinesq hypothesis) through an eddy-viscosity coefficient. Two additional transport equations have to be solved, one for the kinetic energy and one for the dissipation per unit turbulence kinetic energy, to compute the local values of the eddy viscosity. The $\mathrm{k}-\omega$ model is more accurate then $\mathrm{k}-\bar{E}$ model in the near wall layers, but it fails for flows with pressure induced separation. In addition, $\omega$ equation shows strong sensitivity to the values of $\omega$ in the free-stream outside the boundary layer.

\subsection{SST K- $\omega$ TURBULENCE MODEL}


The SST k- $\omega$ turbulence model is a two-equation eddy-viscosity model. SST model formulation (Menter, et al., 2003):

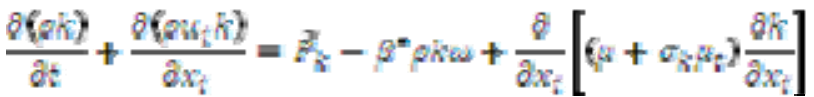

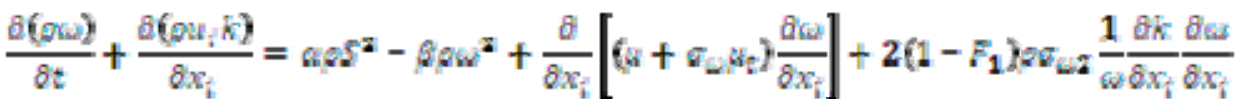

\section{RESULTS}

Each simulation has approximately the same number of grid elements. Also, the same turbulence model was implemented for each simulation. Different barrier configurations were studied. This was done in order to find the most suitable barrier configuration regarding the wind velocity in disembarkation area and the wind load on the barrier and the building. The criterion parameter for optimum barrier configuration is wind velocity to be around $5 \mathrm{~m} / \mathrm{s}$ in disembarkation area.

The first numerical simulation has no barriers, Fig. 7. This is done in order to compare no barrier wind velocity data with wind velocity data for different barrier configuration and show barrier effect on velocity reduction in disembarkation area.

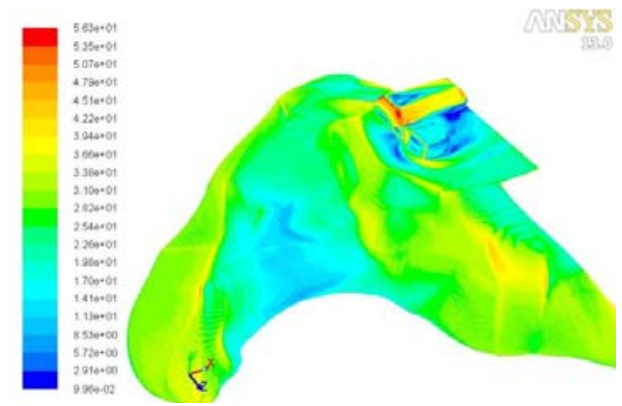

Figure 7. Velocity profile for the first case (no barrier) $(\mathrm{m} / \mathrm{s})$

The first barrier configuration has only one barrier, Fig. 8. The secondbarrier configuration has two barriers, Fig. 9. In particular, the first barrier is larger and further away from the building, and the second barrier is smaller and closer to the building. Finally, the thirdbarrier configuration, Fig. 10, consists of two barriers with the same set up, where the bigger one has different opening configuration than the previous barrier configuration. 


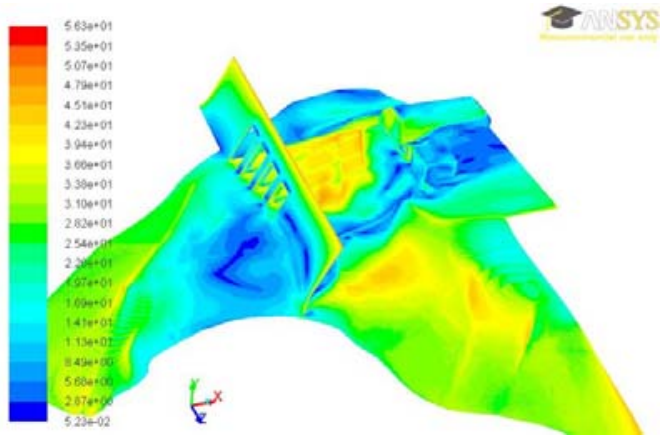

Figure 8. Velocity profile for the second case (one barrier) $(\mathrm{m} / \mathrm{s})$

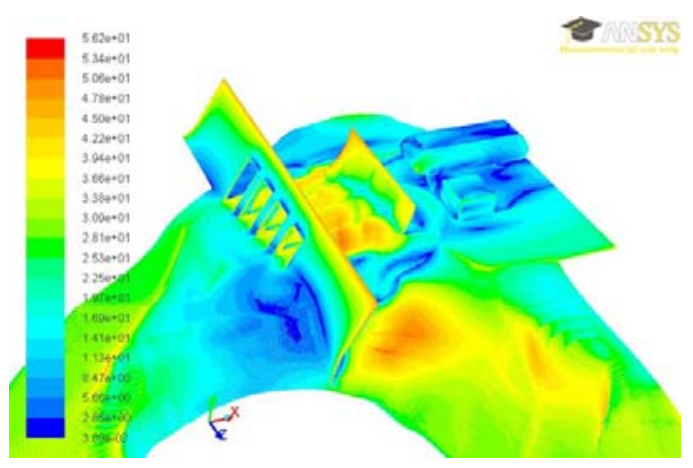

Figure 9. Velocity profile for the third case (two barriers compact) $(\mathrm{m} / \mathrm{s})$

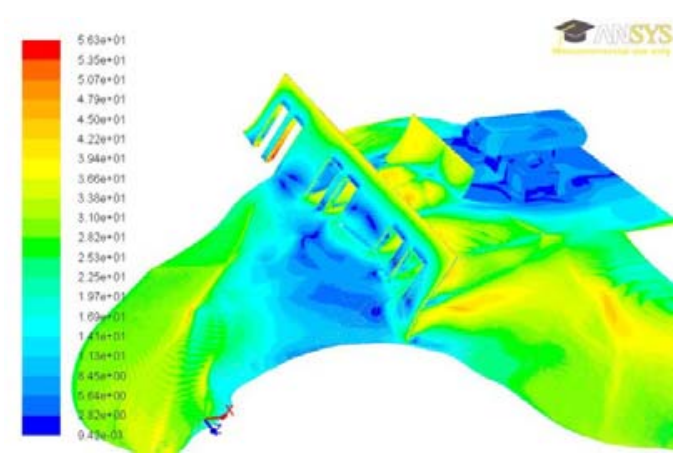

Figure 10. Velocity profile for the fourth case (two barriers spread) $(\mathrm{m} / \mathrm{s})$

One can notice that the velocity is lower in disembarkation area for the three barrier configurations. Data collections were taken along a sampling line 0 -x, shown in Fig. 11. Also, in the Fig. 11 is shown disembarkation area.

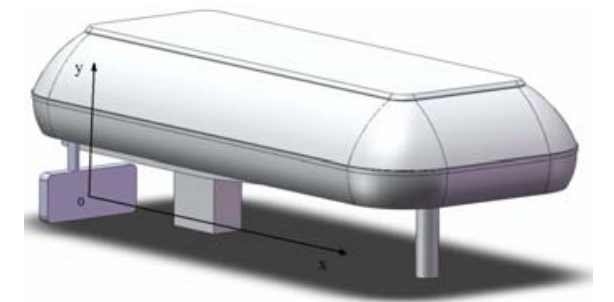

Figure 11. Sampling line position at the disembarkation area

Two measurements were taken for each simulation, first measurement for velocity magnitude, and the second measurement, streamwise component of the velocity. Wind direction is front, normal to the building for all these cases.

Comparison among all four cases are shown for velocity magnitude and streamwise component of the velocity, Fig. 12 and Fig. 13, respectively. Zero position is in regard to position of the seat in Fig. 11. 


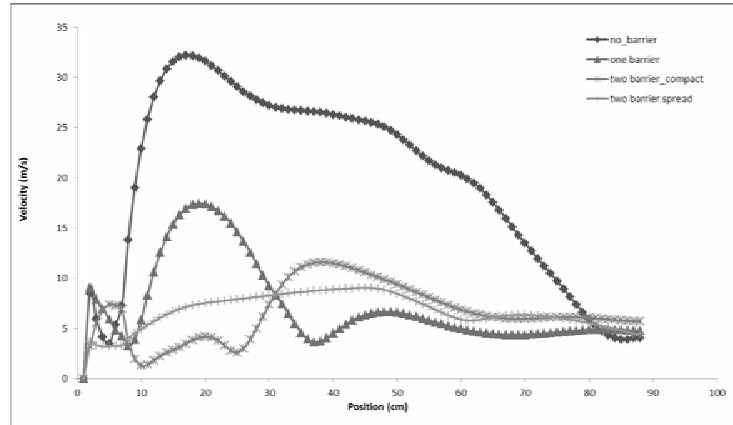

Figure 12: Velocity magnitude for four barrier cases

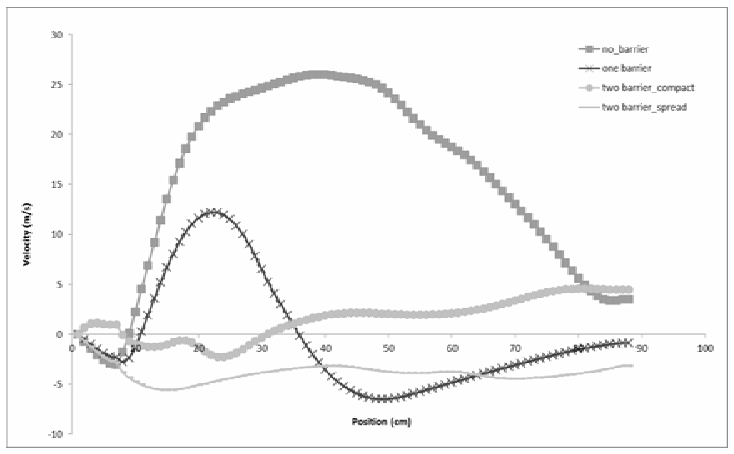

Figure 13: Streamwise component of the velocity for four barrier cases

Wind velocity without the barrier is around $30 \mathrm{~m} / \mathrm{s}$ in disembarkation area. Ski lift normal function is obstructed in this high wind velocity condition. There is an obvious need for a wind barrier which will lower the wind velocity in disembarkation area. Three different barrier configurations were considered.

First barrier configuration, with one barrier, has noticeably lower wind velocity compared to the case without a barrier. However, with the first barrier configuration, the wind velocity is not sufficiently low to satisfy the criterion for normal function of the ski-lift, which is around $5 \mathrm{~m} / \mathrm{s}$.

One can notice from Fig.13 that the second and the third barrier configuration, two barrier_compact and two barrier_spread, have lower wind velocity comparing to the first barrier configuration in disembarkation area. Wind velocity for these two configurations is around $5 \mathrm{~m} / \mathrm{s}$. Both barrier configurations satisfy the velocity criteria. However, two barrier_spread configuration is more suitable since the wind load on the barrier for this configuration is lower due to the arrangement of the openings on the walls.

Also, one can notice from Fig. 13 that the streamwise component of the velocity is negative in few positions. Moreover, for the last configuration, streamwise component of the velocity is only negative. Negative value of the velocity streamwise component means that the direction of the wind in disembarkation area is opposite of the incoming wind direction. This is beneficial since opposite direction of the approaching wind is the same direction of incoming ski lift seat. Hence, wind does not interrupt the normal function of the ski lift.

From the three barrier configurations, the last configuration has both, that is the low wind velocity and low wind load on the barrier. Furthermore, streamwise component of the velocity in the boarding area is negative. Therefore, the last configuration is the most suitable for ski lift wind protection in severe wind conditions.

\section{CONCLUSION}

Wind flow over hill, barrier and building was successfully numerically simulated. Velocity field was very well predicted. Four numerical simulations were run on Prelog, super computer at Faculty of Mechanical Engineering in Ljubljana. Barrier influence on the wind velocity in disembarkation area was analyzed with each numerical simulation. Different barrier configurations were analyzed. CAD model of the hill, building and the barrier was created in 
SolidWorks, grid was generated with Ansys CutCell Mesher, and Ansys Fluent was use as a solver. Turbulence model used in CFD simulation is shear stress transport (SST) $k-w$ model.

All three barrier configurations produced lower wind velocity in disembarkation area. The last two barrier configurations produced wind velocity which satisfies the criterion for wind velocity in disembarkation area. Moreover, the last barrier configuration satisfies the barrier wind load criterion. Wind velocities in the disembarkation area with the wind barriers are much smaller than the initial $40 \mathrm{~m} / \mathrm{s}$ velocity. This allows normal usage of the ski lift in severe wind conditions. The goal of this research is accomplished, that is, barrier configuration is defined where 5 to 6 $(\mathrm{m} / \mathrm{s})$ wind velocity in disembarkation area is produced.

\section{REFERENCES}

[1] N. N. Sorensen, A Bechmann, J Johansen, L Myllerup, P Botha, S Vinther, B S Nielsen "Identification of severe wind conditions using a Raynolds Averaged Navier-Stokes solver" The Science of Making Tourque from Wind, Journal of Physics: Conference Series 75 (2007) 012053

[2] Andreas Bechmann Large-Eddy Simulation of Atmospheric Flow over Complex Terrain, Riso-PhD-28(EN), Riso National Lab., Roskilde, Denmark, 2006

[3] Jackson P.S., Hunt J.C.R. Turbulent Wind Flow Over a Low Hill, Quart. J.R. Met. Soc., 101:929-955. 1975

[4] F. A. Castro, J. M. L. M. Palma and A. Silva Lopes Simulation of the Askervein flow. PART 1: Reynolds averaged navier-stokes equations ( $k=\omega$ turbulence model)

[5] Eric Berge, Arne R. Gravdahl, Jan Schelling, Lars Tallhaug, Ove UndheimWind in complex terrain. A comparison of WAsP and two CFD-models.

[6] Pingzhi Fang, Ming Gu, Jianguo Tan, Bingke Zhao and Demin Shao Modeling the neutral atmosheric boundary layer based on the standard $\mathrm{k}-\varepsilon$ turbulent model: modified wall function.

[7] T. K. Guha, R. N. Sharma, P.J. Richards "CFD modeling of wind induced mean and fluctuating external pressure coefficients on the Texas Technical University building”, EACWE 5, 19-23 July 2009 